\title{
Pituitary Apoplexy After Intravitreal Injection of Vascular Endothelial Growth Factor Inhibitor: A Novel Complication
}

\author{
Rebecca A. Kasl ${ }^{1}$ Heather M. Kistka ${ }^{1}$ Justin H. Turner ${ }^{2}$ Jessica K. Devin ${ }^{3}$ Lola B. Chambless ${ }^{4}$
}

${ }^{1}$ Department of Neurosurgery, Vanderbilt University Medical Center, Nashville, Tennessee, United States

2 Department of Otolaryngology-Head and Neck Surgery, Vanderbilt University School of Medicine, Nashville, Tennessee, United States

${ }^{3}$ Division of Diabetes, Endocrinology and Metabolism, Department of

Address for correspondence Rebecca A. Kasl, MD, Department of Neurosurgery, Vanderbilt University Medical Center, T4224 Medical Center North, Nashville, TN 37232, United States Medicine, Vanderbilt University Medical Center, Nashville, Tennessee, United States

${ }^{4}$ Division of Neurological Surgery, Department of Neurosurgery, Vanderbilt University Medical Center, Nashville, Tennessee, United States

J Neurol Surg Rep 2015;76:e205-e210.

\begin{abstract}
Keywords

- pituitary apoplexy

- hemorrhage

- vascular endothelial growth factor inhibitor

- ranibizumab

Pituitary adenomas are common in the general population. They can be complicated by intratumoral hemorrhage, otherwise known as apoplexy, which frequently presents with neurologic deficits that may necessitate urgent surgical decompression. Many risk factors for pituitary apoplexy have been suggested in the literature. We present a case of symptomatic apoplexy in a woman following the intravitreal administration of the vascular endothelial growth factor (VEGF) inhibitor ranibizumab. Ophthalmoplegia resolved and visual acuity significantly improved following gross total resection of the tumor via an endoscopic endonasal surgical approach. The association between intravitreal injection of a VEGF inhibitor and pituitary apoplexy has not been previously described, but physicians performing these procedures should be aware of this potential complication.
\end{abstract}

\section{Introduction}

Pituitary adenomas may present with a gradual decline in vision, cranial nerve palsies, or change in endocrine function. Less commonly, an average of $2 \%$ of surgically treated pituitary adenomas present acutely with intratumoral hemorrhage, termed apoplexy. ${ }^{1,2}$ Previously described triggers of this rare presentation include trauma, antithrombotic therapy, coagulopathy, recent surgical intervention, dopamine agonists, and essential hypertension. ${ }^{3-7}$ Clinically, apoplexy is characterized by vision loss, ophthalmoplegia, headache, or nausea, and it often warrants urgent surgical intervention. ${ }^{1}$

received

September 5, 2014

accepted

April 9, 2015

published online

August 24, 2015
Vascular endothelial growth factor (VEGF) is an important regulator of tumor angiogenesis. Increased VEGF expression is associated with hemorrhage in pituitary adenomas. ${ }^{8,9}$ Due to the critical role of VEGF in tumor growth, intravenous infusion of VEGF inhibitors is increasingly used in neurooncology to treat refractory high-grade neoplasms. ${ }^{10,11}$ Data from these patients have revealed an increased risk of intratumoral hemorrhage with this treatment. ${ }^{12}$ VEGF inhibitors are also used in ophthalmology to treat a variety of conditions believed to stem from increased angiogenesis including diabetic retinopathy, macular degeneration, and central serous chorioretinopathy. ${ }^{13,14}$ Intraocular hemorrhage after intravitreal injection of VEGF inhibitors has been described. ${ }^{15,16}$
License terms

Stuttgart · New York

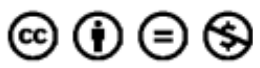


We present the first reported case of pituitary apoplexy after intravitreal injection of the VEGF inhibitor ranibizumab. Prompt recognition of this event and urgent surgical resection of the lesion in this case provided this patient with an excellent functional outcome.

\section{Case Report}

\section{History}

A 74-year-old woman presented to neurosurgical attention with acute left-sided unilateral vision loss and complete left oculomotor nerve palsy. She had received an intravitreal ranibizumab injection and fluorescein angiography 2 days prior for a new diagnosis of central serous retinopathy.

The patient initially presented to the ophthalmology clinic with a few months of worsening blurry vision in her left eye. Her ophthalmologic history was significant for cataracts, hyperopia, and astigmatism requiring eyeglasses. There was no history of trauma, ocular surgery, or pituitary tumor. She had no prior intracranial imaging. Her medical history was notable for primary hypothyroidism and essential hypertension managed with levothyroxine and verapamil. In the clinic, her corrected visual acuity was significantly worse in her left eye (20/200) than in her right (20/30), and intraocular pressures were normal. Neurologic examination was unremarkable with equal, round, and reactive pupils bilaterally, intact extraocular movements, and full visual fields to confrontation. Slit lamp examination was normal. Her fundal examination was only notable for a $1.5-\mathrm{mm}$ left choroidal nevus. Optical coherence tomography demonstrated chronic idiopathic central serous chorioretinopathy (left more than right).

Intravitreal injection of ranibizumab was recommended to treat central serous chorioretinopathy. The treatment was administered by a retinal specialist in the clinic later that day. Prior to the ranibizumab injection, fluorescein corneal angiography was performed without complication. Ranibizumab was then injected at the 4:00 site on the left eye. The patient tolerated the procedure well and went home in stable condition.

Forty-seven hours later, the patient was seen emergently in the ophthalmology clinic. She relayed a history of diffuse headache and neck stiffness beginning the day after the procedure. Symptoms progressively worsened, peaking on the morning of presentation when she awoke with left ptosis, diplopia, persistent headache, and nausea. Upon examination in clinic, she was alert and oriented. Her right eye examination was unchanged, but her left visual acuity had acutely worsened from 20/200 to 20/400 with normal intraocular pressure. She demonstrated left ptosis and a fixed and dilated left pupil at $6 \mathrm{~mm}$. Her left eye was laterally and inferiorly deviated with impaired medial adduction and impaired left consensual response when exposed to light on the right. Slitlamp and dilated fundus examinations were unchanged. She was emergently transferred to the emergency department (ED) with a diagnosis of acute left oculomotor nerve palsy.

In the ED, a noncontrast computed tomography (CT) scan of the head revealed a sellar mass with leftward hemorrhagic expansion and bony erosion (-Fig. 1). CT angiography was negative. Follow-up magnetic resonance imaging (MRI) exhibited acute hemorrhage within a $17 \times 25 \times 25 \mathrm{~mm}$ mass compressing the optic chiasm and invading the left cavernous sinus consistent with pituitary apoplexy (-Fig. 2). Pituitary function tests demonstrated central hypothyroidism superimposed on preexisting primary hypothyroidism (thyroidstimulating hormone [TSH] $0.128 \mathrm{mIU} / \mathrm{L}$, free thyroxine [FT4] $0.88 \mathrm{ng} / \mathrm{dL}$ ), and central hypogonadism in a postmenopausal woman (luteinizing hormone $[\mathrm{LH}] 0.4 \mathrm{mIU} / \mathrm{mL}$, follicularstimulating hormone [FSH] $3.7 \mathrm{mIU} / \mathrm{mL}$ ). Prolactin was normal at $6.0 \mathrm{ng} / \mathrm{mL}$. After neurosurgical evaluation and endocrine consultation, the patient was administered dexamethasone and scheduled for urgent endoscopic transsphenoidal resection.

\section{Operation}

The patient was placed under general anesthesia, positioned supine, and administered preoperative dexamethasone and antibiotics. A neurosurgeon and rhinologist collaborated for the procedure, and the BrainLAB (Munich, Germany) stereotactic navigation system guided their approach. Bilateral endoscopic sphenoidotomies and a posterior septectomy were performed to create a common cavity. The sella turcica was opened widely to expose the dura, which was incised in a cruciate fashion. Hemorrhagic pituitary tumor was recognized immediately and resected circumferentially. Numerous specimens were obtained and sent for permanent pathology. An intraoperative cerebrospinal fluid leak was noted and repaired with a Durepair (Medtronic, Minneapolis, Minnesota, United States) underlay graft and free mucosal overlay graft harvested from the left middle turbinate. The repair was supported with dry Gelfoam (Pfizer, New York, United States) and gloved Merocel (Medtronic, Minneapolis, Minnesota,

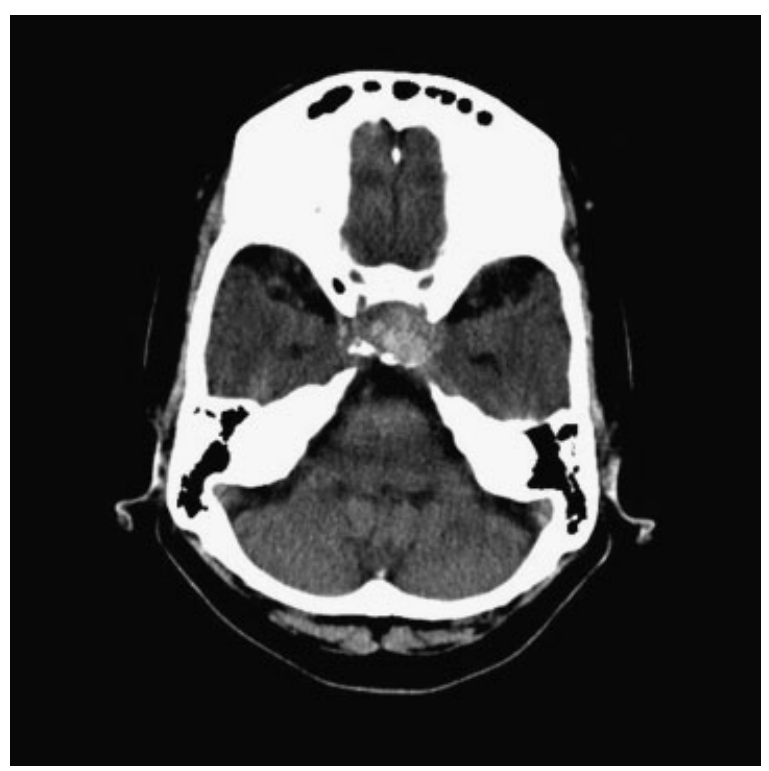

Fig. 1 Noncontrast computed tomography of the head demonstrating hemorrhage within the sella with surrounding bony erosion consistent with pituitary apoplexy. 

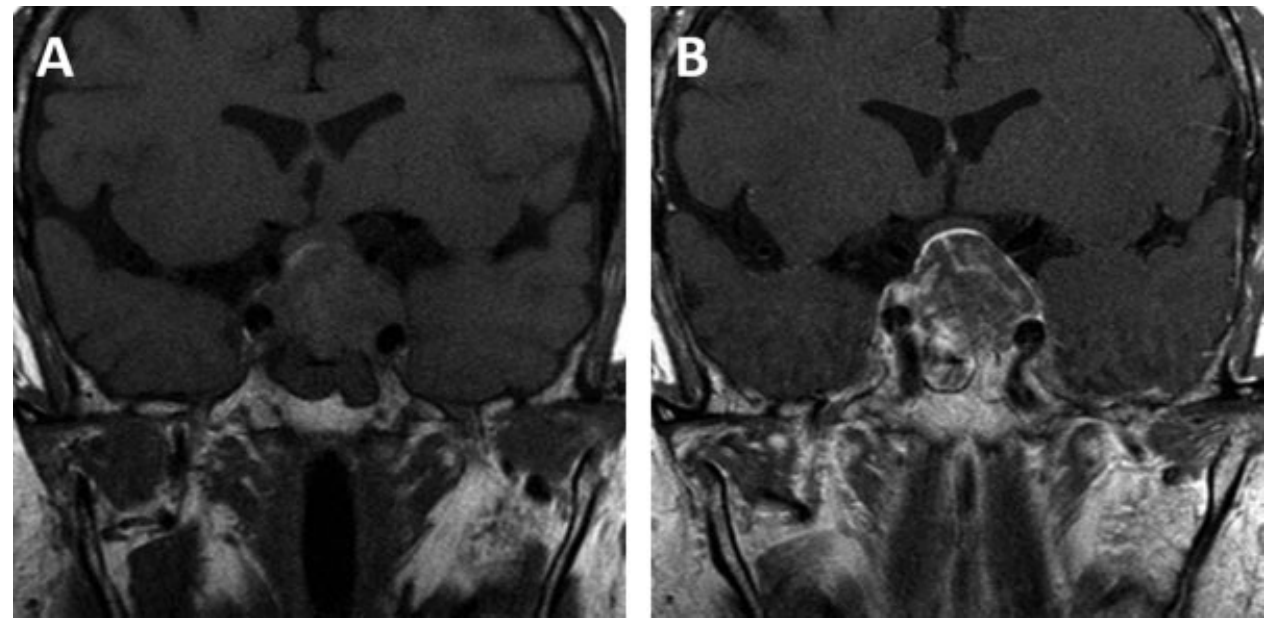

Fig. 2 (A) Precontrast and (B) postcontrast T1-weighted magnetic resonance imaging of the brain demonstrating a $17 \times 25 \times 25 \mathrm{~mm}$ pituitary mass with evidence of hemorrhage, chiasmal compression, and cavernous sinus invasion.

United States) sponges. The patient awoke from anesthesia without difficulty and was taken to the neurologic intensive care unit in stable condition.

\section{Pathologic Findings}

Histologic sections showed small foci of viable pituitary adenoma characterized by cells with monomorphic roundto-ovoid nuclei arranged in perivascular palisades. Large regions of tumor demonstrated incipient cell death with loss of nuclear detail and increased cytoplasmic eosinophilia, and areas of frankly necrotic tumor were observed. Abundant hemorrhage and an acute inflammatory infiltrate were seen. Tumor cells showed immunoreactivity for synaptophysin, FSH, and LH. This pathologic description was consistent with a silent gonadotroph, or nonfunctioning, pituitary adenoma (-Fig. 3).

\section{Postoperative Course}

On postoperative day (POD) 1, the patient's subjective vision had notably improved. Her corrected left eye visual acuity was tested and noted to be 20/100. By POD 2, her vision was 20/30 and 20/25 with correction in the left and right eyes, respectively. Her intraocular pressure was normal, and pupils were reactive bilaterally. Left-sided ptosis and ophthalmoplegia were improved, but some diplopia persisted. She did not experience any disorders of water metabolism, including diabetes insipidus or hyponatremia, in the postoperative period. Perioperative corticosteroids were tapered to a physiologic replacement dose of $5 \mathrm{mg}$ prednisone daily until her endocrine follow-up appointment. Her levothyroxine dose was optimized following a low normal FT4 laboratory result. She was discharged to home on POD 3, by which point her ptosis and ophthalmoplegia had nearly recovered.

Her follow-up period was uneventful. At her 6-week follow-up appointment, MRI illustrated gross total resection with complete decompression of the optic apparatus and no evidence of recurrent tumor (-Fig. 4). She had complete resolution of her oculomotor nerve palsy and believed her vision was better than it had been in years. Her endocrine laboratory evaluation confirmed panhypopituitarism
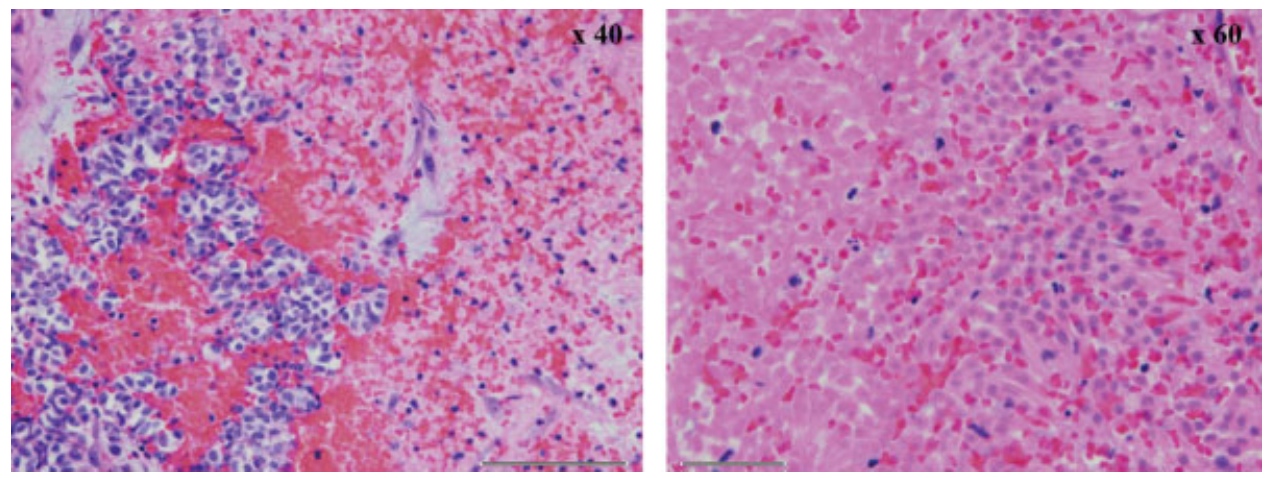

Fig. 3 The pathologic specimen at $\times 40$ and $\times 60$ magnification. At $\times 40$ : A small focus of viable neoplasm (left side of image) is surrounded by hemorrhage, necrosis, and infiltrating leukocytes (hematoxylin and eosin [H\&E], original magnification $\times 400$; scale bar $=100$ microns). At $\times 60$ : Spectrum of adenoma cell death with loss of nuclear detail and cytoplasmic hypereosinophilia (right side of image) progressing to frank necrosis (left) (H\&E, original magnification $\times 600$; scale bar $=50$ microns). 


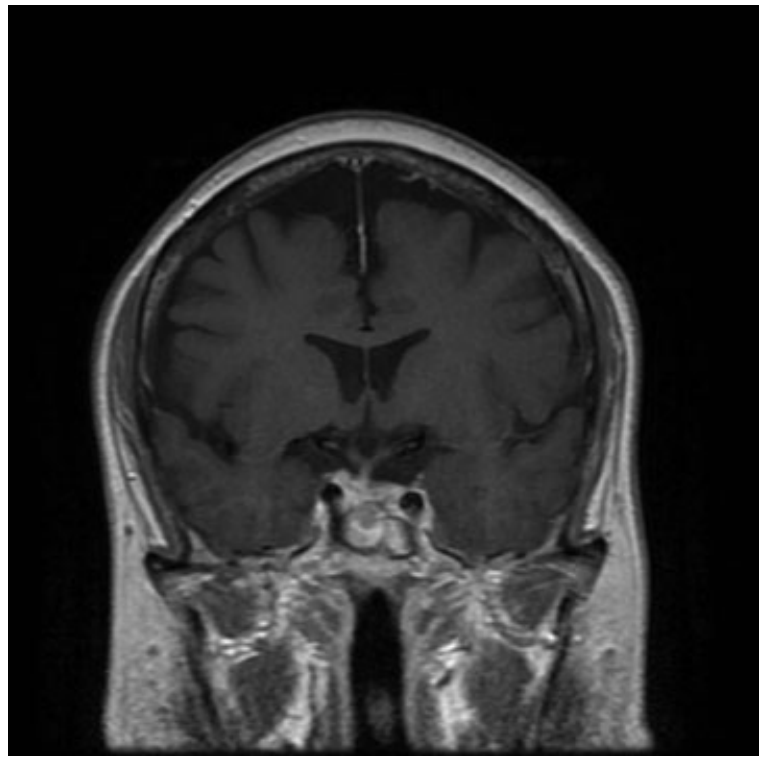

Fig. 4 A 6-week postoperative postcontrast T1-weighted magnetic resonance imaging of the brain demonstrated surgical decompression of the optic apparatus and infundibulum without evidence of residual tumor.

(baseline adrenocorticotropic hormone $6 \mu \mathrm{g} / \mathrm{dL}$ and cortisol $2.4 \mu \mathrm{g} / \mathrm{dL}$ with a stimulated cortisol of $13.9 \mu \mathrm{g} / \mathrm{dL} 1$ hour after $250 \mu \mathrm{g}$ Cortrosyn, TSH 0.246 mIU/mL, FT4 1.17 ng/dL, LH 1.4 $\mathrm{mIU} / \mathrm{mL}$, and FSH $4.6 \mathrm{mIU} / \mathrm{mL}$ ). She remains on treatment for central adrenal insufficiency and hypothyroidism. Postoperative neuro-ophthalmology examination demonstrated 20/20 and 20/70 corrected vision in the right and left eyes, respectively, with full and equal peripheral vision via Goldmann visual fields. The examination confirmed a visually significant cataract in her left eye and concomitant macular degeneration. Because delayed recurrence following apoplexy is not uncommon, she will be followed with annual pituitary MRIs to monitor for any evidence of tumor recurrence. $^{17}$

\section{Discussion}

Pituitary adenomas are common intracranial tumors. ${ }^{18}$ They are typically benign, and a third are nonfunctional. Although nonfunctional tumors lack hormone production, they often stain positively for $\mathrm{LH}$ and $\mathrm{FSH}^{19,20}$ Given the endocrine inactivity, their course is typically occult until a macroadenoma becomes large enough to compress neighboring structures such as the optic chiasm and surrounding pituitary tissue. As such, the most common symptomatic presentation includes gradual onset of vision loss, hormonal deficiencies, or headache.

Pituitary apoplexy is defined as acute hemorrhage or infarction of the pituitary mass causing rapid expansion of the sella turcica. Pituitary apoplexy can be a challenging diagnosis because patients frequently have no history of an adenoma. The classic acute clinical presentation of apoplexy involves headache (63-100\%), visual deficits (40-100\%), and nausea/vomiting (59-78\%). ${ }^{1,21-23}$ Laboratory work-up usual- ly demonstrates hypopituitarism (88\%), ${ }^{1,21}$ and the diagnosis is confirmed by intracranial imaging.

Although the precise pathophysiologic mechanism for apoplexy has not been ascertained, rapid tumor growth exceeding or compressing the vascular supply as well as primary hemorrhagic or ischemic events has been proposed. ${ }^{1,24}$ As a tumor grows, so does its vascular supply. In fact, the pituitary gland's robust vasculature contributes to a 5.4-fold greater chance of hemorrhage when compared with other central nervous system tumors. ${ }^{25}$ Additionally, nonfunctional adenomas and prolactinomas carry a higher incidence of apoplexy. ${ }^{26}$ Thus our patient's large nonfunctioning macroadenoma possessed several risk factors for hemorrhage including tumor size and type, essential hypertension, and recent surgical intervention.

Pituitary apoplexy following injection of an intravitreal VEGF inhibitor has not been previously described in the literature. VEGF is a protein that promotes angiogenesis. As such, overexpression of VEGF is associated with tumor growth and retinal vascular disease progression. Inhibitors of VEGF, such as the monoclonal antibody bevacizumab (Avastin) and antibody derivative ranibizumab (Lucentis), have an evolving therapeutic role in retinopathy by targeting the neovascularization and vascular permeability processes inherent to the disease pathogenesis. ${ }^{27}$

VEGF upregulation also has a proposed role in pituitary hemorrhage. ${ }^{9}$ Elevated VEGF expression has been reported in hemorrhagic pituitary tumors, regardless of an apoplectic clinical presentation. $8,28,29$ This patient received localized therapy, yet intravitreal injections of VEGF have demonstrated widespread complications indicating systemic distribution of the drug. ${ }^{30,31}$ One hypothetical pathophysiologic mechanism behind apoplexy is the medication-induced apoptosis of endothelial cells. ${ }^{32}$ VEGF is known to maintain endothelial stability. ${ }^{33}$ The acute perturbation in the endothelial lining could precipitate hemorrhage or hemorrhagic infarction in a pituitary adenoma, a tumor type known for its hypervascularity. Although the role of VEGF in spontaneous apoplexy is not fully understood, the VEGF inhibitor ranibizumab likely triggered apoplexy in our patient, which may suggest that her preexisting level of VEGF could have been a significant factor.

Treatment for pituitary apoplexy is surgical resection or conservative therapy. In either case, initial management is to stabilize the patient whose propensity for hormone deficiencies may compromise a surgical intervention. A conservative method often involves high-dose corticosteroids, dopamine agonists for prolactinomas, or radiotherapy. ${ }^{22,34,35}$ Expectant management is considered appropriate for patients without severe visual compromise or altered mental status. ${ }^{2,26,36}$

Preserving vision is the primary goal of surgical intervention for pituitary apoplexy, and outcomes depend on symptom severity and time until intervention.,37 Patients presenting with monocular blindness have a better visual recovery rate than those with binocular blindness. ${ }^{2}$ No difference in visual recovery for patients who underwent surgery within the first day of apoplexy versus 2 to 7 days has been demonstrated. ${ }^{2,38}$ However, early surgery within a week of 
the sentinel event produces better visual outcomes..$^{23,39}$ Our patient's apoplectic attack included severe monocular visual decline; thus urgent surgical management was appropriately pursued. The attack's estimated onset was 1.5 days prior to presentation and 2.5 days prior to surgical intervention. Therefore, her timely presentation to medical care increased her likelihood for visual recovery after tumor resection. Our patient's vision improved quickly to 20/25 and 20/30 in the right and left eyes, respectively, by POD 2 .

Even when vision is saved, pituitary apoplexy is commonly complicated by long-term hypopituitarism. After surgical intervention, hormone replacement is required in $82 \%$ of patients who present with apoplexy. ${ }^{1}$ Diabetes insipidus is a transient complication in 10 to $18 \%$ of cases. ${ }^{1,40}$ On average, long-term glucocorticoids, thyroid hormone, and gonadal steroids are required in $75 \%, 75 \%$, and $95 \%$ of patients, respectively. ${ }^{1}$ Our postmenopausal patient continued to require corticosteroids and thyroid hormone 4 months after her initial presentation.

\section{Conclusions}

We describe a case of pituitary apoplexy after intravitreal injection of a VEGF inhibitor in a 74-year-old woman. A VEGF inhibitor triggering an apoplectic event has not been previously described in the literature. Physicians performing intravitreal VEGF inhibitor injection should be aware of this potential complication because immediate recognition of apoplexy may be key to preserving vision. Although pituitary adenomas are less common than retinopathy in the elderly population, nonfunctional tumors should be considered during evaluation of progressive vision loss. Furthermore, an acute change in vision or focal neurologic deficit warrants rapid imaging. Our patient demonstrated remarkable visual recovery after expeditious surgical intervention with minimal visual acuity deficits and no cranial nerve deficits at 6-week follow-up. Rapid assessment, diagnosis, and treatment were paramount to her recovery.

\section{Acknowledgment}

The authors would like to thank their respective departments at Vanderbilt University Medical Center for their support of this project.

\section{References}

1 Nawar RN, AbdelMannan D, Selman WR, Arafah BM. Pituitary tumor apoplexy: a review. J Intensive Care Med 2008;23(2): 75-90

2 Turgut M, Ozsunar Y, Başak S, Güney E, Kir E, Meteoğlu I. Pituitary apoplexy: an overview of 186 cases published during the last century. Acta Neurochir (Wien) 2010;152(5):749-761

3 Chang CV, Felicio AC, Toscanini AC, Teixeira MJ, Cunha-Neto MB. Pituitary tumor apoplexy. Arq Neuropsiquiatr 2009;67(2A): 328-333

4 Chng E, Dalan R. Pituitary apoplexy associated with cabergoline therapy. J Clin Neurosci 2013;20(12):1637-1643
5 Knoepfelmacher M, Gomes MC, Melo ME, Mendonca BB. Pituitary apoplexy during therapy with cabergoline in an adolescent male with prolactin-secreting macroadenoma. Pituitary 2004;7(2):83-87

6 Randeva HS, Schoebel J, Byrne J, Esiri M, Adams CB, Wass JA. Classical pituitary apoplexy: clinical features, management and outcome. Clin Endocrinol (Oxf) 1999;51(2):181-188

7 Semple PL, Jane JA Jr, Laws ER Jr. Clinical relevance of precipitating factors in pituitary apoplexy. Neurosurgery 2007;61(5):956-961; discussion 961-962

8 Jin Kim Y, Hyun Kim C, Hwan Cheong J, Min Kim J. Relationship between expression of vascular endothelial growth factor and intratumoral hemorrhage in human pituitary adenomas. Tumori 2011;97(5):639-646

9 Xiao Z, Liu Q, Mao F, Wu J, Lei T. TNF- $\alpha$-induced VEGF and MMP-9 expression promotes hemorrhagic transformation in pituitary adenomas. Int J Mol Sci 2011;12(6):4165-4179

10 Lou E, Peters KB, Sumrall AL, et al. Phase II trial of upfront bevacizumab and temozolomide for unresectable or multifocal glioblastoma. Cancer Med 2013;2(2):185-195

11 Gilbert MR, Dignam JJ, Armstrong TS, et al. A randomized trial of bevacizumab for newly diagnosed glioblastoma. $\mathrm{N}$ Engl J Med 2014;370(8):699-708

12 Friedman HS, Prados MD, Wen PY, et al. Bevacizumab alone and in combination with irinotecan in recurrent glioblastoma. J Clin Oncol 2009;27(28):4733-4740

13 Kamba T, McDonald DM. Mechanisms of adverse effects of antiVEGF therapy for cancer. Br J Cancer 2007;96(12):1788-1795

$14 \mathrm{Kim} \mathrm{M}$, Lee SC, Lee SJ. Intravitreal ranibizumab for acute central serous chorioretinopathy. Ophthalmologica 2013;229(3): 152-157

15 Fasih U, Shaikh N, Rahman A, Sultan S, Fehmi MS, Shaikh A. A oneyear follow-up study of ocular and systemic complications of intravitreal injection of bevacizumab (Avastin). J Pak Med Assoc 2013;63(6):707-710

16 Brouzas D, Koutsandrea C, Moschos M, Papadimitriou S, Ladas I, Apostolopoulos M. Massive choroidal hemorrhage after intravitreal administration of bevacizumab (Avastin) for AMD followed by contralateral sympathetic ophthalmia. Clin Ophthalmol 2009; 3:457-459

17 Pal A, Capatina C, Tenreiro AP, et al. Pituitary apoplexy in nonfunctioning pituitary adenomas: long term follow up is important because of significant numbers of tumour recurrences. Clin Endocrinol (Oxf) 2011;75(4):501-504

18 Ostrom QT, Gittleman H, Farah P, et al. CBTRUS statistical report: Primary brain and central nervous system tumors diagnosed in the United States in 2006-2010. Neuro Oncol 2013;15(2, Suppl 2): ii1-ii56

19 Saeger W, Lüdecke DK, Buchfelder M, Fahlbusch R, Quabbe HJ, Petersenn S. Pathohistological classification of pituitary tumors: 10 years of experience with the German Pituitary Tumor Registry. Eur J Endocrinol 2007;156(2):203-216

20 Yamada S, Ohyama K, Taguchi M, et al. A study of the correlation between morphological findings and biological activities in clinically nonfunctioning pituitary adenomas. Neurosurgery 2007; 61(3):580-584; discussion 584-585

21 Leyer C, Castinetti F, Morange I, et al. A conservative management is preferable in milder forms of pituitary tumor apoplexy. J Endocrinol Invest 2011;34(7):502-509

22 Ayuk J, McGregor EJ, Mitchell RD, Gittoes NJ. Acute management of pituitary apoplexy-surgery or conservative management? Clin Endocrinol (Oxf) 2004;61(6):747-752

23 Bills DC, Meyer FB, Laws ER Jr, et al. A retrospective analysis of pituitary apoplexy. Neurosurgery 1993;33(4):602-608; discussion 608-609

24 Semple PL, De Villiers JC, Bowen RM, Lopes MB, Laws ER Jr. Pituitary apoplexy: do histological features influence the clinical presentation and outcome? J Neurosurg 2006;104(6):931-937 
25 Wakai S, Yamakawa K, Manaka S, Takakura K. Spontaneous intracranial hemorrhage caused by brain tumor: its incidence and clinical significance. Neurosurgery 1982;10(4):437-444

26 Bujawansa S, Thondam SK, Steele C, et al. Presentation, management and outcomes in acute pituitary apoplexy: a large singlecentre experience from the United Kingdom. Clin Endocrinol (Oxf) 2014;80(3):419-424

27 Kim R. Introduction, mechanism of action and rationale for antivascular endothelial growth factor drugs in age-related macular degeneration. Indian J Ophthalmol 2007;55(6):413-415

28 Lee JS, Park YS, Kwon JT, Nam TK, Lee TJ, Kim JK. Radiological apoplexy and its correlation with acute clinical presentation, angiogenesis and tumor microvascular density in pituitary adenomas. J Korean Neurosurg Soc 2011;50(4):281-287

29 Arita K, Kurisu K, Tominaga A, et al. Relationship between intratumoral hemorrhage and overexpression of vascular endothelial growth factor (VEGF) in pituitary adenoma. Hiroshima J Med Sci 2004;53(2):23-27

30 Nuzzi R, Tridico F. Local and systemic complications after intravitreal administration of anti-vascular endothelial growth factor agents in the treatment of different ocular diseases: a fiveyear retrospective study. Semin Ophthalmol 2015;30(2): 129-135

31 Pellé G, Shweke N, Duong Van Huyen JP, et al. Systemic and kidney toxicity of intraocular administration of vascular endothelial growth factor inhibitors. Am J Kidney Dis 2011;57(5): 756-759
32 Zangari M, Fink LM, Elice F, Zhan F, Adcock DM, Tricot GJ. Thrombotic events in patients with cancer receiving antiangiogenesis agents. J Clin Oncol 2009;27(29):4865-4873

33 Kuenen BC. Analysis of prothrombotic mechanisms and endothelial perturbation during treatment with angiogenesis inhibitors. Pathophysiol Haemost Thromb 2003;33(Suppl 1):13-14

34 Maccagnan P, Macedo CL, Kayath MJ, Nogueira RG, Abucham J. Conservative management of pituitary apoplexy: a prospective study. J Clin Endocrinol Metab 1995;80(7):2190-2197

35 McFadzean RM, Doyle D, Rampling R, Teasdale E, Teasdale G. Pituitary apoplexy and its effect on vision. Neurosurgery 1991; 29(5):669-675

36 Sibal L, Ball SG, Connolly V, et al. Pituitary apoplexy: a review of clinical presentation, management and outcome in 45 cases. Pituitary 2004;7(3):157-163

37 Chuang CC, Chang CN, Wei KC, et al. Surgical treatment for severe visual compromised patients after pituitary apoplexy. J Neurooncol 2006;80(1):39-47

38 Onesti ST, Wisniewski T, Post KD. Clinical versus subclinical pituitary apoplexy: presentation, surgical management, and outcome in 21 patients. Neurosurgery 1990;26(6):980-986

39 Agrawal D, Mahapatra AK. Visual outcome of blind eyes in pituitary apoplexy after transsphenoidal surgery: a series of 14 eyes. Surg Neurol 2005;63(1):42-46; discussion 46

40 Nemergut EC, Zuo Z, Jane JA Jr, Laws ER Jr. Predictors of diabetes insipidus after transsphenoidal surgery: a review of 881 patients. J Neurosurg 2005;103(3):448-454 